\title{
Importance of gut microbiome regulation for the prevention and recovery process after SARS-CoV-2 respiratory viral infection (Review)
}

\author{
NUROH NAJMI ${ }^{1,2}$, IMAM MEGANTARA ${ }^{2,3}$, LOVITA ANDRIANI $^{4}$, \\ HANNA GOENAWAN ${ }^{2,5,6}$ and RONNY LESMANA ${ }^{2,5,6}$ \\ ${ }^{1}$ Department of Oral Biology, Faculty of Dentistry; ${ }^{2}$ Division of Biological Activty Central Laboratory; \\ ${ }^{3}$ Department of Biomedical Sciences, Faculty of Medicine; ${ }^{4}$ Faculty of Animal Husbandry; \\ ${ }^{5}$ Department of Biomedical Sciences, Physiology Division, Faculty of Medicine, Universitas Padjadjaran; \\ ${ }^{6}$ Center of Excellence in Higher Education for Pharmaceutical Care Innovation, Bandung, West Java 45363, Indonesia
}

Received September 20, 2021; Accepted December 9, 2021

DOI: $10.3892 /$ br.2022.1508

\begin{abstract}
Infection with severe acute respiratory syndrome coronavirus 2 (SARS-CoV-2) has been reported to affect organs other than the lungs, including the liver, brain, kidneys and intestine, and gastrointestinal symptoms, such as nausea, vomiting, diarrhea and abdominal discomfort, have also been reported. Thus, SARS-CoV-2 could potentially directly or indirectly regulate the gut microbiome profile and its homeostasis. The abundance of Coprobacillus, Clostridium ramosum and Clostridium are associated with the severity of COVID-19, and Firmicutes, Bacteriodetes, Proteobacteria and Actinobacteria are also related to COVID-19 infection. The four phyla are correlated with the severity of COVID-19 infection in patients. The modulation of factors that control the physiological growth of the gut microbiome will determine the proportionate ratio of microbiome types (profile). Taken together, gut microbiome profile alterations in COVID-19 patients may have a cross effect with the modulation of cytokine levels in COVID-19 infection. With these findings, several factors that regulate gut microbiome homeostasis may support the degree
\end{abstract}

Correspondence to: Dr Ronny Lesmana, Department of Biomedical Sciences, Physiology Division, Faculty of Medicine, Universitas Padjadjaran, Bandung, West Java 45363, Indonesia E-mail: ronny@unpad.ac.id

Abbreviations: ACE-2, angiotensin converting enzyme 2; COVID-19, coronavirus disease-19; HCQ, hydroxychloroquine; IL, interleukin; GI, gastrointestinal; MERS-CoV, Middle East respiratory syndrome coronavirus; RNA, ribonucleic acid; SARS-CoV-2, severe acute respiratory syndrome coronavirus 2; SCFAs, short-chain fatty acids; TNF- $\alpha$, tumor necrosis factor- $\alpha$; HDI, herb-drug interaction; AZ, azithromycin

Key words: microbiome, dysbiosis, SARS-CoV-2, supplement, herbal, exercise of the clinical symptoms and hasten the recovery process after COVID-19 infection.

\section{Contents}

1. Introduction

2. Literature search

3. Microbiome profile and COVID-19

4. Modulation of the microbiome profile to regulate the inflammatory response

5. Conclusions

\section{Introduction}

COVID-19 is a disease caused by the severe acute respiratory syndrome coronavirus 2 (SARS-CoV-2), which was first documented in Wuhan, China. This disease has similarities with viral pneumonia and symptoms similar to those of SARS-CoV and MERS-CoV. A total of 3-6 days after infection, upper or lower respiratory tract symptoms, fever and diarrhea, or a combination of these, appear (1). Kang et al (2) reported that $45.8 \%$ of COVID-19 patients not only have respiratory symptoms, but also gastrointestinal (GI) symptoms, such as lack of appetite, diarrhea, vomiting and abdominal pain. OF 9,696 patients, 988 were reported to exhibit nausea and vomiting. Meanwhile, loss of appetite and abdominal pain were reported in 20 and 27 studies, respectively. Loss of appetite and other GI symptoms have also been found in other cases, for example in the UK (3). In cases of reinfection with the SARS-CoV-2 variant B.1.1.7, the symptoms and duration were similar; however, reinfection was rare (4).

Patients with GI tract symptoms have been reported to feel pain for a longer period of time than those without GI tract symptoms. These patients also have higher mean liver enzyme levels, lower monocyte counts, longer prothrombin times and require additional antimicrobial treatment (5). Intestinal dysfunction results in changes in intestinal microbes and increases the levels 
of inflammatory cytokines (5). There are several means by which SARS-CoV-2 can damage the GI tract. First, the virus can damage the GI tract via an inflammatory response. Second, it directly damages the intestinal mucosa and causes disturbances in the GI system. Third, it can induce changes in the composition of the intestinal flora, causing digestive disorders. The modification in the composition of intestinal flora causes respiratory disorders through the 'gut-lung axis' (6).

In patients with comorbidities, the immune system is affected, and the severity of COVID-19 infection worsens. One common comorbidity in children and adults is obesity, which can compromise the function of several organs and biological systems due to excessive adipose tissue, a deficit in lean mass, insulin resistance, dyslipidemia, hypertension, high levels of pro-inflammatory cytokines and low levels of essential nutrients. Furthermore, obesity is not only associated with the immune, respiratory, cardiovascular and urinary systems, but can also modify the gut microbiota (dysbiosis) $(7,8)$. Obese patients also have longer hospitalization periods, and have higher levels of C-reactive protein and lower lymphocyte counts, which are considered as early indicators of a more severe COVID-19 infection course (9). Another factor that can worsen COVID-19 infection is diabetes, which was shown by the high percentage of ICU admissions among diabetics and a longer hospitalization period, although diabetes was not associated with mortality (10). It was shown that the most common symptoms of COVID-19 patients with diabetes were fever, dry cough, and fatigue (11). Li et al (12) reported that COVID-19 infection caused ketosis or ketoacidosis and induced diabetic ketoacidosis in diabetic patients, which prolonged hospitalization and increased mortality (12).

Infection with COVID-19 might affect the microbiome profile, which is associated with the abundance of some microbiome phylum. Khan et al (13) reported that several phyla related to COVID-19 infection included Firmicutes, Bacteroidetes, Proteobacteria and Actinobacteria, which are also associated with the severity of COVID-19 infection. The correlation between the gut microbiome and COVID-19 infection is interesting. In this review, the link between COVID-19 and the gut microbiome, as well as its potential clinical application in the future is discussed.

\section{Literature search}

A total of 160 articles from PubMed and Google Scholar were initially obtained; 40 articles were excluded as they did not match the research theme. The key words relevant for this review were: 'gut microbiota COVID-19,' 'gastrointestinal tract COVID-19,' 'gut-lung-axis COVID-19,' 'probiotics microbiome COVID-19,' 'drugs COVID-19,' and 'exercise microbiome.' Articles written in English published between 2011 and 2021 as well as relevant articles from the reference lists were retrieved and reviewed. A flow chart showing the literature review process is shown in Fig. 1.

\section{Microbiome profile and COVID-19}

The composition of the gut microbiome changes during a mild, moderate or severe infection, and when a person recovers from infection (14). The abundance of Coprobacillus,
Clostridium ramosum and Clostridium is associated with the severity of COVID-19. In addition, there was an inverse correlation between the abundance of Faecalibacterium prausnitzii (anti-inflammatory bacteria) and the severity of the disease (Table I). Kim et al (15) reported that the abundance of Firmicutes was positively correlated with the severity of COVID-19 symptoms. However, Firmicutes-negative patients have also been reported. Moreira-Rosário et al (16) reported that the ratio of Firmicutes and Bacteroidetes decreased in COVID-19 patients admitted to the ICU from ambulatory wards. The diversity of the gut microbiome affects the condition of patients with COVID-19. According to Ren et al (17), Faecalibacterium has anti-inflammatory properties and has been recognized as a potential biomarker of human health (17). According to Zuo et al (14), COVID-19 patients exhibit changes in the composition of the microbiome compared with healthy people, which results in an increased probability of being infected with opportunistic pathogens during hospitalization. In other respiratory infections, such as with the respiratory syncytial virus, microbiota diversity is associated with weight loss. Viral respiratory infections can lead to reduced intake of nutrients that can alter the gut microbiota (18). In similar cases, Middle East Respiratory Syndrome (MERS) is associated with the gut microbiome. Proteobacteria were more abundant in fatal cases than in non-fatal cases, including species from the Acinetobacter and Klebsiella genera. Patients infected with 2009 influenza A H1N1 pneumonia were found to have increased numbers of Proteobacteria compared with non-viral cases of pneumonia (19). However, information on the detailed mechanism of COVID-19 infection on bacteria in the intestine and the function of the intestine itself is sparse. Several factors can affect homeostasis in the gut, and genetic factors that can affect the gut microbiota cannot be excluded. The present study revealed the possible regulatory effects of COVID-19 on microbiota homeostasis that may impact a patient's health. In this review, the possibility of modulating the balance of gut microbiota through various factors, such as exercise, nutrition-probiotics, prebiotics, vitamins, drugs and herbal medicines are discussed, and these may be considered as future treatment modalities to hasten recovery of COVID-19 patients. The microbiome profile associated with COVID-19 effects are summarized in Table I.

Pathophysiology of viral infections on the effects of the gut microbiota. There are many variants of the SARS-CoV-2 virus, and all have specific genetic markers associated with changes that can result in increased transmissibility or virulence, reduced neutralization by antibodies acquired through infection or vaccination, and increased ability to evade detection or decreased effectiveness of therapy or vaccination (20). SARS-CoV-2 infects human lungs, but other studies have highlighted the vulnerability of other organs, including the liver, brain, kidneys and intestine, to SARS-CoV-2 infection (21). Common symptoms of SARS-CoV-2 infection include a cough, nausea, vomiting and diarrhea followed by abdominal discomfort (22-24) Patients with mild respiratory symptoms accompanied by GI symptoms can experience rapid progression of infection (25). The gastric, duodenal and rectal epithelium showed positive immunofluorescent staining of the viral host receptor and the SARS-CoV-2 viral 


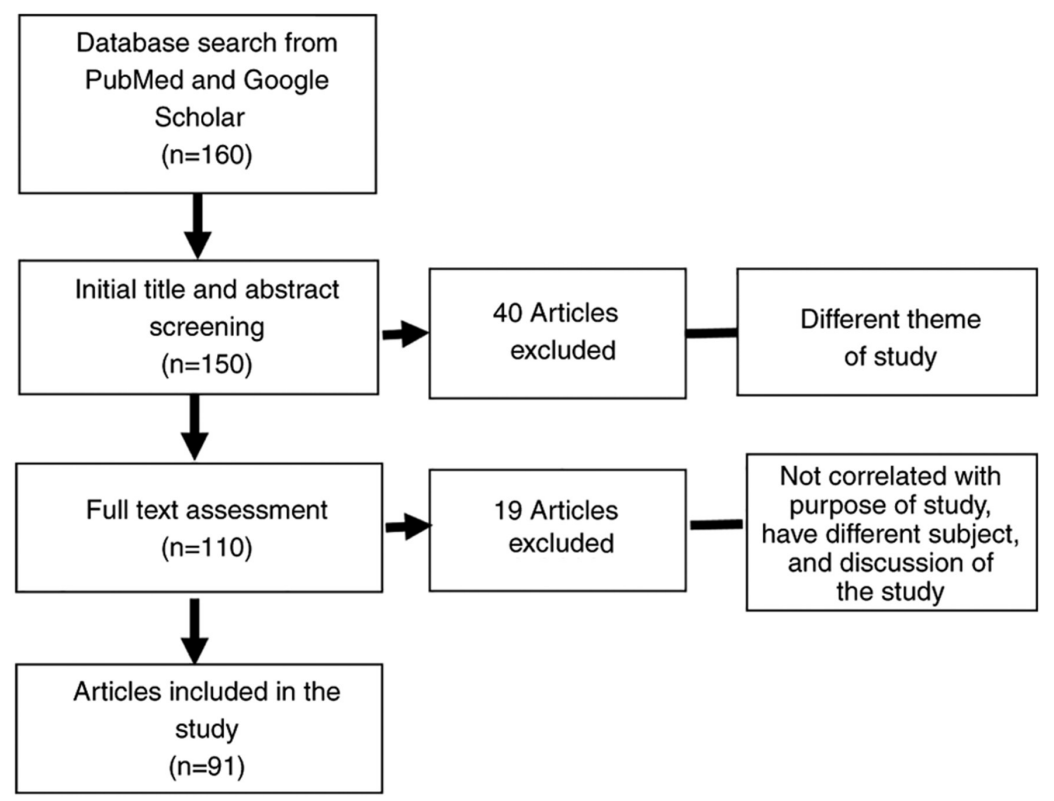

Figure 1. Flow chart of the literature review process.

protein nucleocapsid. In addition, evidence has shown that GI SARS-CoV-2 infection has the potential for fecal-oral transmission (26).

SARS-CoV-2 enters our body via the lung parenchyma. The angiotensin converting enzyme 2 (ACE-2) receptor, which is widely expressed in endocrine organs, including the testes, pancreas, thyroid, adrenal and pituitary glands, is used by coronaviruses as the route of entry into the host cell (27). ACE-2 expression is highest in erythrocytes and is also expressed in the small intestine, large intestine and duodenum (25). SARS-CoV-2 is associated with ACE-2, which can also be expressed by fat. Obesity can inhibit macrophage activation and migration, impair the generation of neutralizing antibodies and memory $\mathrm{T}$ cells, and decrease the activation of effector cells of the immune system, hence its correlation with chronic inflammation (28). In addition, type 2 diabetes mellitus can increase ACE- 2 expression in the lungs and other tissues due to the hyperglycemic response. Hyperglycemia can lead to alveolar-capillary microangiopathy and non-enzymatic protein glycation in the lungs. This makes collagen less susceptible to proteolysis, which causes collagen accumulation in connective tissues in the lungs, resulting in restrictive lung disease (29). The role of ACE-2 is essential for numerous organs, including the lungs, kidneys and gut. ACE-2 participates in the degradation of digestive enzymes that yields free amino acids and influences bacterial metabolism, generating bioactive peptide fragments that include angiotensin ACE-2 (30,31). ACE-2 possesses RAS-independent functions that regulate intestinal amino acid homeostasis, antimicrobial peptide expression, and gut microbiome ecology. ACE-2 is also a key regulator of dietary amino acid homeostasis, innate immunity, gut microbial ecology and infectious susceptibility to colitis (31).

Yang et al (32) reported that the gut microbiota is important for the regulation of colonic ACE-2 expression. Additionally, it may contribute to the gut-lung axis pathology during COVID-19 (32). The human gut microbiota has important roles in human physiology, including maintenance of the epithelial barrier, inhibition of adhesion of pathogens to the intestinal surface, modulation of the maturation of the immune system and degradation of indigestible carbons, such as plant polysaccharides and metabolite production (33). Humans naturally obtain gut microbiota from the mother during breastfeeding. Thereafter, microbiota are obtained from food intake and the environment (34). A healthy gut microbiota is rich in Bifidobacterium spp., Faecalibacterium spp., Ruminococcus spp. and Prevotella spp. $(35,36)$. The dominant bacterial phyla found in the intestine are Firmicutes and Bacteroidetes (37). The intestinal barrier epithelium has important roles; apart from being protective, it also acts as a reservoir for host-produced antimicrobial molecules, such as secretory and defensive IgA. There are host-microbial interactions that are regulated through host recognition of microbe-associated molecular patterns and bacterial metabolites; thus, if there are changes in the host conditions and the microbiome composition, this will contribute to differences in mucus composition and increased susceptibility to infection (38).

Gut microbiome manipulation to alter the gut-lung axis may potentially protect humans against respiratory infections. The gut-lung axis can be described as a common mucosal immunological system. There is a correlation between the gut and lung, particularly with regard to respiratory immune and anti-infective responses. In COVID-19, the gut can downregulate the expression of ACE-2 in the intestine. Probiotics may have potential use for reducing upper respiratory tract infection symptoms (39). The gut and respiratory tract are associated with the modulation of the immune response and dysbiosis. There is microbial translocation with the decrease in intestinal barrier integrity followed by a secondary infection. Chronic lung diseases can develop along with GI tract diseases (40). Genetic or exogenous factors, including diets and antibiotic treatment, can alter the composition of the gut microbiota and can result in a reduced capacity to mount adequate local and 


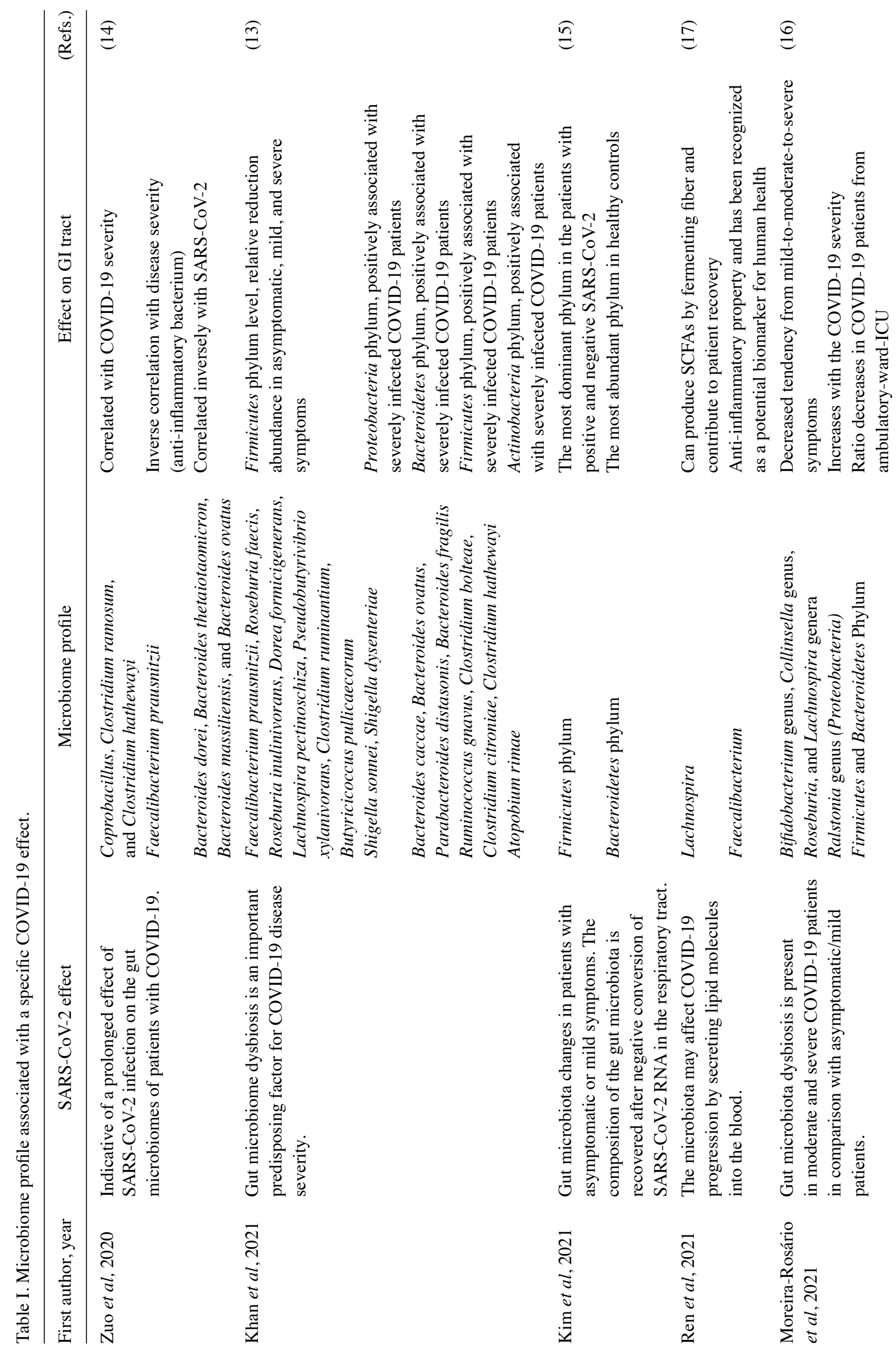


systemic immune responses. The gut microbe-derived components and metabolites include short-chain fatty acids (SCFAs) associated with the gut-lung axis. The direct effects of SCFAs is accomplished through the engagement of $\mathrm{G}$ protein-coupled receptors (41).

Microbiome interaction and cytokine regulation. There is an interaction between the microbiome and cytokines, and this host-microbiome interaction is associated with the production of tumor necrosis factor- $\alpha$ (TNF- $\alpha$ ) and interferon (IFN)- $\gamma$. If there is a change in the composition of the microbiome in the digestive tract, the protective mechanism will be disrupted, leading to an increase in the risk of infection. The gut microbiome plays a role in homeostasis (42). Given the importance of the gut microbiota in the immune response, and knowing that SARS-CoV-2 progression appears to be associated with the 'cytokine storm', which leads to hyper-inflammation (elevated levels of pro-inflammatory cytokines, including TNF- $\alpha$, IL- 6 and IL-1 $\beta$ ), a particular focus should be given to the gut microbiota $(20,27)$. The increase in cytokine levels is associated with severe COVID-19. In addition, the levels of leukocytes, lymphocytes and IL-6 are also increased (40). Excessive IL-6 signaling leads to a myriad of biological effects that contribute to organ damage, such as inducing vascular endothelial growth factor expression in epithelial cells and increasing vessel permeability (43).

The gut microbiota and cytokines are correlated with each other due to butanoate metabolism and butyrate production (44). Butyrate is involved in the regulation of various inflammatory factors, including IL-10, IL-6 and IL-18 (44). In a study by Blanco-Melo (45), it was reported that there was a host response to viral infection. This response was determined by common type I and III IFNs coupled with increased chemokines and high IL-6 expression. SARS-CoV-2 also induces the levels of chemokines. Lin et al (46) reported that certain cytokines were associated with the gut microbiome in patients with Parkinson's Disease (46). In that study, a positive correlation between the abundance of Bacteroides genus and the plasma concentration of TNF- $\alpha$ in Parkinson's disease was identified. Microbial metabolites regulate the host immune system. Bacteroidetes and Clostridium produce SCFAs and desaminotyrosine, which can enhance influenza-specific CD8+ T-cell functions and type I IFN signaling in macrophages, increasing protection against influenza infection (47).

\section{Modulation of the microbiome profile to regulate the inflammatory response}

Exercise. During the pandemic, working from home resulted in changes in food intake and physical activity. According to research by Brancaccio et al (48), participation in physical activity decreased during the pandemic. However, since physical activity has several beneficial effects, certain individuals performed exercises by utilizing internet-based platforms, such as social media (48). Physical activity can help alter the composition of the gut microbiota (48). Quiroga et al (49) reported that in obese children who were physically active, changes in the microbiome significantly reduced Proteobacteria levels, which tended to be similar to those found in healthy children. 
In addition, there is an interaction between the microbiota and host cell immunity (49). Toll-like and Nod-like receptor are signaling pathways responsible for a state of low-grade inflammation, and their expression levels are increased during physical exercise and obesity (49).

Exercise can affect the gut integrity and the composition of gut microbiota in the host. In a study by Campbell et al (50), comparisons between groups of animals showed the presence of a diverse range of gut microbiota (50). However, Clostridiales dominated the fecal microbiota present in all animal groups. Faecalibacterium prausnitzii was detected only in animals who exercised, whereas animals with typical diets without exercise, had large clusters of Lachnospiraceae spp., which were not present in animals fed high-fat diets. Allobaculum spp. and Clostridium spp. were found in animals with regular diets who exercised, whereas animals fed high-fat diets had microbial groups associated with Peptococcus spp (50).

In addition, a study of rugby athletes by Clarke et al (51) showed significant differences between the athletes and control group. The athletes had higher taxa microbiota: 22 phyla, 68 families and 113 genera. Exercise and protein intake increased gut microbiota diversity in the athletes. Firmicutes and Ruminococcaceae were abundantly present, whereas Bacteroidetes were less abundant in the athletes (51). During exercise, TNF receptors gradually produce TNF- $\alpha$ inhibitors, the levels of which increase during and after exercise. Similarly, IL-1 receptor antagonists are produced during exercise and remain elevated post-exercise (52). Physical exercise acts as a modulator of the immune system as it can increase pro- and anti-inflammatory cytokines and secular lymphocytes, and IFN-I can promote macrophage and lymphocyte activity. Suppression of the IFN-I response has been noted in COVID-19. In addition, severe COVID-19 infections have been associated with a pro-inflammatory cytokine storm, lymphopenia, circulatory changes and viral spread to other organs (53).

\section{Nutrition}

Probiotics. Probiotics are non-pathogenic microbes that are beneficial to humans. The most common species are Lactobacillus and Bifidobacterium, which are present in several fermented foods $(54,55)$. Probiotics provide protection as they have a low disease activity index and cause considerably little histopathological damage in the large intestine. In addition, probiotics are more effective in modulating the host immune response, as they decrease IL-1 $\beta$ and IL-6 levels and increase the expression of TGF- $\beta$ and IL-10 (56). Consumption of Lactobacillus reuteri V3401 can reduce IL-6 and sVCAM levels (57). Probiotics have been reported to increase the levels of IFN-I and the number and activity of antigen-presenting cells, natural killer cells and T-cells, as well as systemic and mucosa-specific antibodies in the lungs. Clinical evidence indicates that modulation of the gut microbiota can positively influence COVID-19 disease progression (58).

Fermented milk and yogurt are the most common consumed forms of probiotics. In a study by McNulty et al (59), probiotic bacterial strains were shown to affect the gut microbiota. There were no significant changes in the bacterial composition or representation of gene-encoding enzymes after consuming fermented milk (59). However, the results of fecal sample sequencing and urine metabolite spectrometry showed significant changes in the expression of microbiome-encoded enzymes involved in various metabolic pathways, the most prominent of which were related to carbohydrate metabolism. Bacterial strains present in fermented milk or yogurt were not associated with the abundance of these phyla microbiota in the gut. There were differences in Bifidobacterium strains in individuals who consumed fermented milk mixed with probiotic strains and Bifidobacterium probiotics compared to Lactobacillus-fermented milk. Fermented probiotic milk and yogurt intake were associated with the levels of ingested bacteria (60). The composition of different bacteria in probiotic products can affect the environment of the gut microbiota, and the interaction between the gut microbiota and host will provide protection against viral infections. Probiotics provide protection, treatment and prevention of illness in the digestive system.

Prebiotics. Consumption of prebiotics is a dietary strategy that can modify the GI microbiota for health benefits (61). Prebiotics are substrates selectively utilized by host microorganisms that confer a health benefit. Prebiotics enhance the growth of Bifidobacteria and Lactobacilli, which have beneficial effects on system-wide metabolism and physiology. Prebiotics are predominantly carbohydrate-based, but other substances, such as polyphenols and polyunsaturated fatty acids, may exert prebiotic effects. Bacteroides, one of the microbiome genera, can break down high molecular weight polysaccharides (62).

Prebiotics are fibers that cannot be digested by the host but are metabolized by the colonic microbiome, resulting in the growth of certain bacteria. Intervention with prebiotics, probiotics, or synbiotics is important, as it can alter the composition of gut microbiota in the GI tract. This is essential for the development of appropriate immune regulatory networks, which influences disease risk later in life (63). Dietary consumption of certain food products can result in significant changes in the gut microbiota composition. The change in Bifidobacteria may be attributed to prebiotics (64). The concept of prebiotics is based on increasing the presence of beneficial microorganisms in the gut microbiota, such as Bifidobacteria and Lactobacilli. Since the diversity of the gut microbiota has expanded, there may be other potential beneficial genera, such as Roseburia, Eubacterium, Faecalibacterium, Akkermansia, Christensenella and Propionibacteria (58). Prebiotics, probiotics, fermented foods and synbiotics can modulate the gut and extend its benefits to distant sites, such as the respiratory tract (65).

Vitamins. COVID-19 is associated with vitamin D deficiency. The rate of SARS-CoV-2 infection was significantly lower in vitamin D-deficient patients supplemented with cholecalciferol than those without supplementation (66). According to Robles-Vera et al (67), the abundance of Prevotella and Actinomyces increased, whereas that of Odoribacteraceae and genus Butyricimonas decreased in mice on a vitamin D-free diet. Vitamin D deficiency did not induce intestinal dysbiosis, but it resulted in several specific changes to the bacterial taxa, which might play a pathophysiological role in the immunological dysregulation associated with hypovitaminosis (67). Vitamin D is now recognized as a hormone with several extra-skeletal actions, including in the immune system. 
The microbiome is correlated with the levels of vitamin D, and Firmicutes phylum, Clostridia class and Clostridia order have been recognized as butyrate producers. Some of the genera identified were Ruminococcus, Coprococcus and Blautia obeum species. Specific microbiota, known as butyrate producers, provide potential targets for intervention, either through dietary modification or vitamin D supplementation (68). Vitamin D deficiency is associated with the cytokine storm and an increased likelihood of requiring a ventilator in hospitalized COVID-19 patients. Meanwhile, dexamethasone appears to mitigate the adverse effects of vitamin D deficiency (69).

Vitamin A (VA) exhibits pharmacological activity in the management of pneumonia. The mechanisms of action of VA against SARS-CoV-2 include enrichment of immunoreactions, inhibition of inflammatory reactions and biological processes related to reactive oxygen species (70). VA can assist in promoting intestinal immunity and epithelial integrity (71). According to a study by Liang et al (28), VA may contribute to the clinical management of CHOL/COVID-19 by inducing cell repair, suppressing oxidative stress and inflammatory reactions, and ameliorating immunity (28). One of the roles of $\mathrm{VA}$ is regulating the proliferation of $\mathrm{B}-$ and T-cell differentiation and interaction, including induced T-cell migration (72). VA can affect gut immunity and epithelial integrity, factors that may, in turn, modulate microbiome development. In neonates, VA is associated with a relatively higher abundance of Bifidobacterium, and a good VA status is associated with a higher abundance of other genera, such as Akkermansia (71).

Drug-related. Over the course of a humans life, an individual will be exposed to numerous drugs, including vaccinations, painkillers, antibiotics and contraceptives. COVID-19 treatment requires limiting viral multiplication and neutralizing tissue damage induced by an inappropriate immune reaction (73). The microbiome can act as a drug as it can secrete enzymes with a biocatalyst effect (74). Drugs can affect the composition of the microbiome in terms of abundance, diversity and function. If there is a deviation, then this will result in dysbiosis. Changes in the microbiome due to administration of drugs can be temporary or long-term (74). There is a positive correlation between the quantity of drugs taken and overall microbiome composition (74). Bifidobacterium decreases during multidrug treatment, which in elderly COVID-19 patients, not only causes dysbiosis in the intestine but also increases bacterial resistance, and in adults the dynamic changes to the microbiome was complex during COVID-19 infection (75,76). Antibiotic use (and misuse) and polypharmacy may promote the selection of resistant commensal strains, which constitute a reservoir of transmittable resistance factors in elderly populations with comorbidities (75).

Antibiotic-induced alterations of the gut microbiome, as an ecologically complex system, may also result in metabolic changes in the host, increasing the risk of weight gain and obesity, altering the immune response and increasing susceptibility to other infections from a loss of colonization resistance $(74,77)$. The top microbiome-associated drugs include proton pump inhibitors lipid-lowering statins, laxatives, metformin, $\beta$-blockers, ACE inhibitors and selective serotonin reuptake inhibitors (77).
The uncontrolled inflammatory process is of particular concern in cases of COVID-19. Research by Dormont et al (78) reported that cases of COVID-19 that resulted in death might be caused by virus-induced hyper-inflammation; multidrug nanoparticles (NPs) were the topic of this study. These multidrug NPs enable efficient encapsulation of drugs, reduce side effects and promise anti-inflammatory and protective effects in lethal endotoxemia and systemic shock models (78). The combination of traditional Chinese and Western medicine may carry risks of herb-drug interaction (HDI) (79). A study by Liu et al (79) reported that HDIs primarily occurred in the GI tract, and typically led to diarrhea, but this did not develop or was milder when taking a single drug. According to Dai et al (80), the consistency between the gut microflora and glycosidase system indicates the inhibition of darunavir on the activity of $\beta$-glucosidase and $\beta$-glucuronidase. Regarding darunavir (with a high HDI risk), when patients with COVID-19 use traditional herbal and anti-coronavirus medicines in combination, it is necessary to adjust the intake interval to avoid alteration of the efficacy and toxicity to prevent/mitigate adverse effects (80).

Hydroxychloroquine (HCQ) is an anti-malarial drug that can be used as treatment of COVID-19, and the combination of chloroquine and HCQ is already used in the treatment of COVID-19. Chloroquine does not affect the expression of ACE-2 on cell surfaces, but it inhibits terminal glycosylation of the ACE-2 receptor for cell entry, which is targeted by SARS-CoV and SARS-CoV-2. HCQ can be used as treatment for rheumatoid arthritis (73). Pan et al (81) reported that HCQ did not increase intestinal permeability. The effect of HCQ was detected on gene expression of tight junction-associated proteins in colonic tissue, and there was no statistically significant change in the mRNA expression levels of several claudins (1, 2, 4, 5, 8 and 14), occludin, cadherin 1, mucin 2 and zonula occludens 1 (ZO-1) after HCQ challenge for 14 days. However, HCQ could alter the gut microbiota composition, and the dominant phyla in these groups were Bacteroidetes and Firmicutes (81). Million et al (82) reported that simultaneous intake of HCQ and azithromycin (AZ) could be used as a treatment for COVID-19. Clinical results of a combination of HCQ and AZ reported mild adverse events (GI or skin symptoms, headache, insomnia and transient blurred vision). However, AZ can induce a modest decline in the microbiota and a shift in taxonomic composition driven by a reduction in Proteobacteria and Verrucomicrobia (specifically, Akkermansia muciniphila) (83). Moreover, the combination of $\mathrm{AZ}$ and florfenicol can alter the gut microbiota composition and decrease the abundance and diversity. At the phylum level, the ratio of Firmicutes/Bacteroidetes increased significantly in the antibiotic groups (84). Drug treatment in COVID-19 patients can affect the GI microbiome. However, the gut microbiome can provide efficacy and safety by changing the structure of the drug enzymatically and altering the bioavailability, bioactivity or toxicity of the drug.

Herbal. Herbal medicines have several benefits for the GI tract and can serve as alternative treatments. The Nutrition Care (NC) Gut Relief Formula contains a combination of herbs and nutrients, including curcumin, aloe vera, slippery elm, guar gum, pectin, peppermint oil and glutamine. The NC Gut Relief Formula significantly improved GI tract problems and 


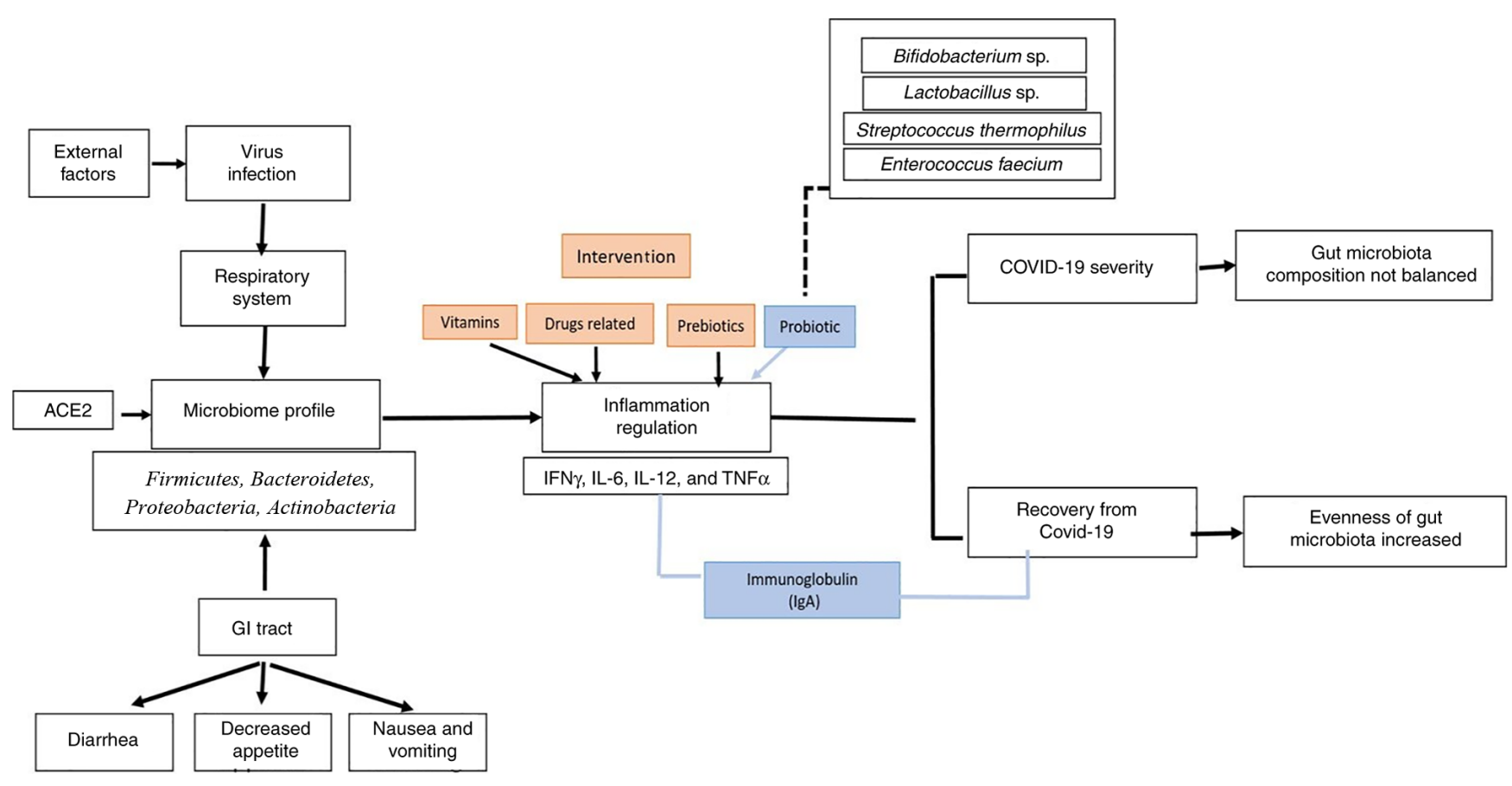

Figure 2. Schematic of microbiome regulation in association with COVID-19 viral infection. ACE-2, angiotensin converting enzyme 2; COVID-19, coronavirus disease-19; IL, interleukin; GI, gastrointestinal; TNF- $\alpha$, tumor necrosis factor- $\alpha$.

the microbiome profile, and reduced or increased inflammation (85). Traditional Chinese herbal medicines have played a vital role in the treatment of SARS, and combined with Western medicines, significantly improve the symptoms of SARS (86). Herbal medicines have fewer side effects than classic pharmaceuticals (87).

Herbal medicine and functional foods contain fiber, polyphenols and polysaccharides, exerting prebiotic-like activities to prevent and treat cardiometabolic diseases (88). The abundance of Bacteroidetes and Akkermansia, Bifidobacteria, Lactobacillus, Bacteroides and Prevotella is increased by herbal medicine and functional foods. A recent study showed that curcumin improved intestinal barrier function by modulating intracellular signaling and organizing tight junctions, providing a mechanism where curcumin modulates chronic inflammatory diseases (88). Peterson et al (89) reported that herbal medicines drove the formation of unique microbial communities. Herbal medicines also induced blooms of butyrate and propionate production.

Herbal medicines can modulate the gut microbiota in a manner predicted to improve colonic epithelium function, reduce inflammation and provide protection from opportunistic bacteria. Herbal medicine, or natural active ingredients, can reduce blood glucose and lipid levels and lower body and visceral adipose weights (90). Herbs can restore the structure of the intestinal floral, and herbs can aid COVID-19 treatment by modulating the immune system. The intestinal flora is closely related to the expression profile of tight junction proteins in the intestinal epithelial cells, including claudin-1, occludin and ZO-1. Five main categories of herbal ingredients can affect the intestinal flora: Glycosides, flavonoids, alkaloids, phenylpropanoids and organic acids. China's guidelines mentioned that herbs and prebiotics can be used in the treatment of COVID-19, especially for maintaining intestinal floral homeostasis and preventing secondary bacterial infections. One of the beneficial effects of herbal based medicines is they inhibit the activity of SARS-CoV-2 3C-like protease (91).

\section{Conclusions}

The modulation of the factors that control the physiological growth of the gut microbiome will determine the proportionate ratio of microbiome types, which is important for modulating cytokine levels that impact inflammatory processes, such as in the case of COVID-19 infection (Fig. 2). This review highlights the potential future directions and clinical applications for subsequent studies. It hypothesized that modulation of the microbiome may hasten the recovery process or reduce the severity of an illness via regulation of the inflammatory process.

\section{Acknowledgements}

The authors would like to thank Mr. Deby Fajar Mardhian (Department of Dental Materials and Technol, Universitas Padjadjaran) who assisted in reviewing and proofreading the manuscript.

\section{Funding}

This study was supported by a WCR grant from the Ministry Education and Culture (grant no. 1207/UN6.3.1/PT.00/2021), and an Academic Leadership grant (grant no. 1959/UN6.3.1/PT.00/2021).

\section{Availability of data and materials}

The datasets used and/or analyzed during the present study are available from the corresponding author on reasonable request. 


\section{Authors' contributions}

NN, IM, LA, HG and RL performed the literature search and assisted in drafting and revising the manuscript. All authors have read and approved the final manuscript. Data authentication is not applicable.

\section{Ethics approval and consent to participate}

Not applicable.

\section{Patient consent for publication}

Not applicable.

\section{Competing interests}

The authors declare that they have no competing interests.

\section{References}

1. Chan JFW, Yuan S, Kok KH, To KK, Chu H, Yang J, Xing F, Liu J, Yip CC, Poon RW, et al: A familial cluster of pneumonia associated with the 2019 novel coronavirus indicating person-to-person transmission: A study of a family cluster. Lancet 395 : 514-523, 2020 .

2. Kang MK, Kim KO, Kim MC, Cho JH, Kim SB, Park JG Kim KH, Lee SH, Jang BI and Kim TN: Clinical characteristics of coronavirus disease 2019 patients with diarrhea in Daegu. Korean J Intern Med 35: 1261-1269, 2020.

3. Tariq R, Saha S, Furqan F, Hassett L, Pardi D and Khanna S: Prevalence and Mortality of COVID-19 patients with gastrointestinal symptoms: A systematic review and Meta-analysis. Mayo Clin Proc 95: 1632-1648, 2020.

4. Graham MS, Sudre CH, May A, Antonelli M, Murray B, Varsavsky T, Kläser K, Canas LS, Molteni E, Modat M, et al: Changes in symptomatology, reinfection, and transmissibility associated with the SARS-CoV-2 variant B.1.1.7: An ecological study. Lancet Public Heal 6: e335-e345, 2021.

5. Cascella M, Rajnik M, Cuomo A, Dulebohn SC and Di Napoli R: Features, evaluation and treatment coronavirus (COVID-19. Review from StatPearls Publishing, Treasure Island (FL), 10 Mar, 2020.

6. Pan L, Mu M, Yang P, Sun Y, Wang R, Yan J, Li P, Hu B, Wang J, Hu C, et al: Clinical characteristics of COVID-19 patients with digestive symptoms in Hubei, China: A descriptive, cross-sectional, multicenter study. Am J Gastroenterol 115: 766-773, 2020

7. Nogueira-de-Almeida CA, Del Ciampo LA, Ferraz IS, Del Ciampo IRL, Contini AA and Ued FDV: COVID-19 and obesity in childhood and adolescence: A clinical review. J Pediatr (Rio J) 95: 546-558, 2020.

8. Yang J, Hu J and Zhu C: Obesity aggravates COVID-19: A systematic review and meta-analysis. J Med Virol 93: 257-261, 2021.

9. Gao F, Zheng KI, Wang XB, Sun QF, Pan KH, Wang TY, Chen YP, Targher G, Byrne CD, George J and Zheng MH: Obesity is a risk factor for greater COVID-19 severity. Diabetes Care 43: e72-e74, 2020.

10. Al-Salameh A, Lanoix JP, Bennis Y, Andrejak C, Brochot E, Deschasse G, Dupont H, Goeb V, Jaureguy M, Lion S, et al Characteristics and outcomes of COVID-19 in hospitalized patients with and without diabetes. Diabetes Metab Res Rev 37: e3388, 2019.

11. Wang F, Yang Y, Dong K, Yan Y, Zhang S, Ren H, Yu X and Shi X: Clinical characteristics of 28 patients with Diabetes and COVID-19 in Wuhan, China. Endocr Pract 26: 668-674, 2020.

12. Li J, Wang X, Chen J, Zuo X, Zhang H and Deng A: COVID-19 infection may cause ketosis and ketoacidosis. Diabetes Obes Metab 22: 1935-1941, 2020.

13. Khan M, Mathew BJ, Gupta P, Garg G, Khadanga S, Vyas AK and Singh AK: Gut dysbiosis and il-21 response in patients with severe covid-19. Microorganisms 9: 1292, 2021.
14. Zuo T, Zhang F, Lui GCY, Yeoh YK, Li AYL, Zhan H, Wan Y, Chung ACK, Cheung CP, Chen N, et al: Alterations in gut microbiota of patients with COVID-19 during time of hospitalization. Gastroenterology 159: 944-955.e8, 2020.

15. Kim HN, Joo EJ, Lee CW, Ahn KS, Kim HL, Park Dong-II and Park SK: Reversion of gut microbiota during the recovery Phase in patients with asymptomatic or mild Covid-19: Longitudinal study. Microorganisms 9: 1237, 2021.

16. Moreira-Rosário A, Marques C, Pinheiro H, Araújo JR, Ribeiro P, Rocha R, Mota I, Pestana D, Ribeiro R, Pereira A, et al: Gut microbiota diversity and C-reactive protein are predictors of disease severity in COVID-19 patients. Front Microbiol 12: 705020, 2021

17. Ren Z, Wang H, Cui G, Lu H, Wang L, Luo H, Chen X, Ren H, Sun R, Liu W, et al: Alterations in the human oral and gut microbiomes and lipidomics in COVID-19. Gut 70: 1253-1265, 2021.

18. Groves HT, Higham SL, Moffatt MF, Cox MJ and Tregoning JS Respiratory viral infection alters the gut microbiota by inducing Inappetence. MBio 11: e03236-19, 2020.

19. Aljabr W, Alruwaili M, Penrice-Randal R, Alrezaihi A, Harrison AJ, Ryan Y, Bentley E, Jones B, Alhatlani BY, AlShahrani D, et al: Amplicon and Metagenomic Analysis of Middle East Respiratory Syndrome (MERS) Coronavirus and the Microbiome in Patients with Severe MERS. mSphere 6: e0021921, 2021

20. Raman R, Patel KJ and Ranjan K: COVID-19: Unmasking emerging SARS-CoV-2 variants, vaccines and therapeutic strategies. Biomolecules 11: 993, 2021

21. Trypsteen W, Van Cleemput J, Snippenberg WV, Gerlo S and Vandekerckhove L: On the whereabouts of SARS-CoV-2 in the human body: A systematic review. PLoS Pathog 16: e1009037, 2020.

22. Holshue ML, DeBolt C, Lindquist S, Lofy KH, Wiesman J, Bruce H, Spitters C, Ericson K, Wilkerson S, Tural A, et al: First Case of 2019 novel coronavirus in the united states. N Engl J Med 382: 929-936, 2020.

23. Guan WJ, Ni ZY, Hu Y, Liang WH, Ou CQ, He JX, Liu L, Shan H, Lei CL, Hui DSC, et al: Clinical characteristics of coronavirus disease 2019 in china. N Engl J Med 382: 1708-1720, 2020.

24. Sultan S, Altayar O, Siddique SM, Davitkov P, Feuerstein JD, Lim JK, Falck-Ytter Y and El-Serag HB; AGA Institute. Electronic address: ewilson@gastro.org: AGA institute rapid review of the gastrointestinal and liver manifestations of COVID-19, Meta-analysis of international data, and recommendations for the consultative management of patients with COVID-19. Gastroenterology 159: 320-334.e27, 2020.

25. Zhang J, Garrett S and Sun J: Gastrointestinal symptoms, pathophysiology, and treatment in COVID-19. Genes Dis 8: 385-400, 2021.

26. Xiao F, Tang M, Zheng X, Liu Y, Li X and Shan H: Evidence for gastrointestinal infection of SARS-CoV-2. Gastroenterology 158: 1831-1833.e3, 2020

27. Clemente-Suárez VJ, Ramos-Campo DJ, Mielgo-Ayuso J, Dalamitros AA, Nikolaidis PA, Hormeño-Holgado A and Tornero-Aguilera JF: Nutrition in the actual covid-19 pandemic. A narrative review. Nutrients 13: 1924, 2021.

28. Liang X, Zhou R, Li Y, Yang L, Su M and Lai KP: Clinica characterization and therapeutic targets of vitamin $\mathrm{A}$ in patients with hepatocholangiocarcinoma and coronavirus disease. Aging (Albany NY) 13: 15785-15800, 2021.

29. Rajpal A, Rahimi L and Ismail-Beigi F: Factors leading to high morbidity and mortality of COVID-19 in patients with type 2 diabetes. J Diabetes 12: 895-908, 2020.

30. Penninger JM, Grant MB and Sung JJY: The role of angiotensin converting enzyme 2 in modulating gut microbiota, intestinal inflammation, and coronavirus infection. Gastroenterology 160: 39-46, 2021

31. Hashimoto T, Perlot T, Rehman A, Trichereau J, Ishiguro H, Paolino M, Sigl V, Hanada T, Hanada R, Lipinski S, et al: ACE2 links amino acid malnutrition to microbial ecology and intestinal inflammation. Nature 487: 477-481, 2012.

32. Yang T, Chakraborty S, Saha P, Mell B, Cheng X, Yeo JY, Mei X, Zhou G, Mandal J, Golonka R, et al: Gnotobiotic rats reveal that gut microbiota regulates colonic mRNA of Ace2, the receptor for SARS-CoV-2 Infectivity. Hypertension 76: e1-e3, 2020.

33. Sánchez B, Delgado S, Blanco-Míguez A, Lourenço A, Gueimonde $\mathrm{M}$ and Margolles A: Probiotics, gut microbiota, and their influence on host health and disease. Mol Nutr Food Res 61: 1600240,2017 
34. Putignani L, Del Chierico F, Petrucca A, Vernocchi P and Dallapiccola B: The human gut microbiota: A dynamic interplay with the host from birth to senescence settled during childhood. Pediatr Res 76: 2-10, 2014

35. Hills RD Jr, Pontefract BA, Mishcon HR, Black CA, Sutton SC and Theberge CR: Gut Microbiome: Profound implications for diet and disease. Nutrients 11: 1613, 2019.

36. Villapol S: Gastrointestinal symptoms associated with COVID-19: Impact on the gut microbiome. Transl Res 226: 57-69, 2020.

37. Lv Y, Qin X, Jia H, Chen S, Sun W and Wang X: The association between gut microbiota composition and BMI in Chinese male college students, as analysed by next-generation sequencing. $\mathrm{Br}$ J Nutr 122: 986-995, 2019.

38. Wiertsema SP, van Bergenhenegouwen $\mathbf{J}$, Garssen $\mathbf{J}$ and Knippels LMJ: The interplay between the gut microbiome and the immune system in the context of infectious diseases throughout life and the role of nutrition in optimizing treatment strategies. Nutrients 13: 886, 202.

39. Mullish BH, Marchesi JR, McDonald JAK, Pass DA, Masetti G, Michael DR, Plummer S, Jack AA, Davies TS, Hughes TR and Wang D: Probiotics reduce self-reported symptoms of upper respiratory tract infection in overweight and obese adults: Should we be considering probiotics during viral pandemics? Gut Microbes 13: 1-9, 2021

40. Aktas B and Aslim B: Gut-lung axis and dysbiosis in COVID-19. Turk J Biol 44: 265-272, 2003.

41. Dang AT and Marsland BJ: Microbes, metabolites, and the gut-lung axis. Mucosal Immunol 12: 843-850, 2019.

42. Taco-Masias AA, Fernandez-Aristi AR, Cornejo-Tapia A, Aguilar-Luis MA, del Valle LJ, Silva-Caso W,Zavaleta-Gavidia V, Weilg P, Cornejo-Pacherres H, Bazán-Mayra J, et al: Gut microbiota in hospitalized children with acute infective gastroenteritis caused by virus or bacteria in a regional Peruvian hospital. PeerJ 8: e9964, 2020.

43. Liu B, Li M, Zhou Z, Guan X and Xiang Y: Can we use interleukin-6 (IL-6) blockade for coronavirus disease 2019 (COVID-19)-induced cytokine release syndrome (CRS)? J Autoimmun 111: 102452, 2020

44. Zhang X, Shi L, Sun T and Guo K: Dysbiosis of gut microbiota and its correlation with dysregulation of cytokines in psoriasis patients. BMC Microbiol 21: 78, 2021.

45. Blanco-Melo D, Nilsson-Payant BE, Liu WC, Uhl S, Hoagland D, Møller R, Jordan TX, Oishi K, Panis M, Sachs D, et al: Imbalanced host response to SARS-CoV-2 drives development of COVID-19. Cell 181: 1036-1045.e9, 2020.

46. Lin CH, Chen CC, Chiang HL, Liou JM, Chang CM, Lu TP, Chuang EY, Tai YC, Cheng C, Lin HY and Wu MS: Altered gut microbiota and inflammatory cytokine responses in patients with Parkinson's disease. J Neuroinflammation 16: 129, 2019.

47. He Y, Wang J, Li F and Shi Y: Main clinical features of COVID-19 and potential prognostic and therapeutic value of the microbiota in SARS-CoV-2 infections. Front Microbiol 11: 1302, 2020.

48. Brancaccio M, Mennitti C, Gentile A, Correale L, Buzzachera CF, Ferraris C, Montomoli C, Frisso G, Borrelli P and Scudiero O Effects of the covid-19 pandemic on job activity, dietary Behaviours and physical activity habits of university population of Naples, federico II-Italy. Int J Environ Res Public Health 18: 1502, 2021.

49. Quiroga R, Nistal E, Estébanez B, Porras D, Juárez-Fernández M, Martínez-Flórez S, García-Mediavilla MV, de Paz JA, González-Gallego J, Sánchez-Campos S and Cuevas MJ: Exercise training modulates the gut microbiota profile and impairs inflammatory signaling pathways in obese children. Exp Mol Med 52: 1048-1061, 2020.

50. Campbell SC, Wisniewski PJ, Noji M, McGuinness LR, Häggblom MM, Lightfoot SA, Joseph LB and Kerkhof LJ: The effect of diet and exercise on intestinal integrity and microbial diversity in mice. PLoS One 11: e0150502, 2016

51. Clarke SF, Murphy EF, O'Sullivan O, Lucey AJ, Humphreys M, Hogan A, Hayes P, O'Reilly M, Jeffery IB, Wood-Martin R, et al: Exercise and associated dietary extremes impact on gut microbial diversity. Gut 63: 1913-1920, 2014.

52. Metsios GS, Moe RH and Kitas GD: Exercise and inflammation. Best Pract Res Clin Rheumatol 34: 101504, 2020.

53. da Silveira MP, da Silva Fagundes KK, Bizuti MR, Starck É Rossi RC and de Resende E Silva DT: Physical exercise as a tool to help the immune system against COVID-19: An integrative review of the current literature. Clin Exp Med 21: 15-28, 2021.

54. Singh K and Rao A: Probiotics: A potential immunomodulator in COVID-19 infection management. Nutr Res 87: 1-12, 2021.
55. Lehtoranta L, Pitkäranta A and Korpela R: Probiotics in respiratory virus infections. Eur J Clin Microbiol Infect Dis 33: $1289-1302,2014$

56. Celiberto LS, Pinto RA, Rossi EA, Vallance BA and Cavallini DCU: Isolation and characterization of potentially probiotic bacterial strains from mice: Proof of concept for personalized probiotics. Nutrients 10: 1684, 2018.

57. Tenorio-Jiménez C, Martínez-Ramírez MJ, Del Castillo-Codes I, Arraiza-Irigoyen C, Tercero-Lozano M, Camacho J, Chueca N, García F, Olza J, Plaza-Díaz J, et al: Lactobacillus reuteri V3401 reduces inflammatory biomarkers and modifies the gastrointestinal microbiome in adults with metabolic syndrome: The PROSIR study. Nutrients 11: 1761, 2019.

58. Walton GE, Gibson GR and Hunter KA: Mechanisms linking the human gut microbiome to prophylactic and treatment strategies for COVID-19. Br J Nutr 126: 219-227, 2021.

59. McNulty NP, Yatsunenko T, Hsiao A, Faith JJ, Muegge BD, Goodman AL, Henrissat B, Oozeer R, Cools-Portier S, Gobert G, et al: The impact of a consortium of fermented milk strains on the gut microbiome of gnotobiotic mice and monozygotic twins. Sci Transl Med 3: 106ra106, 2011.

60. Redondo-Useros N, Gheorghe A, Díaz-Prieto LE, Villavisencio B, Marcos A and Nova E: Associations of probiotic fermented milk (PFM) and yogurt consumption with Bifidobacterium and Lactobacillus components of the gut microbiota in healthy adults. Nutrients 11: 651, 2019.

61. Holscher HD: Dietary fiber and prebiotics and the gastrointestinal microbiota. Gut Microbes 8: 172-184, 2017.

62. Sanders ME, Merenstein DJ, Reid G, Gibson GR and Rastall RA: Probiotics and prebiotics in intestinal health and disease: From biology to the clinic. Nat Rev Gastroenterol Hepatol 16: 605-616, 2019.

63. Frei R, Akdis M and O'Mahony L: Prebiotics, probiotics, synbiotics, and the immune system: Experimental data and clinical evidence. Curr Opin Gastroenterol 31: 153-158, 2015.

64. Costabile A, Deaville ER, Morales AM and Gibson GR: Prebiotic potential of a maize-based soluble fibre and impact of dose on the human gut microbiota. PLoS One 11: e0144457, 2016

65. Antunes AEC, Vinderola G, Xavier-Santos D and Sivieri K: Food research international. Food Res Int 37: 195-197, 2004.

66. Oristrell J, Oliva JC, Casado E, Subirana I, Domínguez D, Toloba A, Balado A and Grau M: Vitamin D supplementation and COVID-19 risk: A population-based, cohort study. J Endocrinol Invest: Jul 17, 2021 (Epub ahead of print).

67. Robles-Vera I, Callejo M, Ramos R, Duarte J and Perez-Vizcaino F: Impact of Vitamin D deficit on the rat gut microbiome. Nutrients 11: 2564, 2019.

68. Thomas RL, Jiang L, Adams JS, Xu ZZ, Shen J, Janssen S, Ackermann G, Vanderschueren D, Pauwels S, Knight R, et al: Vitamin D metabolites and the gut microbiome in older men. Nat Commun 11: 5997, 2020.

69. Wenban C, Heer RS, Baktash V, Kandiah P, Katsanouli T, Pandey A, Goindoo R, Ajaz A, Van den Abbeele K, Mandal AKJ and Missouris CG: Dexamethasone treatment may mitigate adverse effects of vitamin D deficiency in hospitalized Covid-19 patients. J Med Virol 93: 6605-6610, 2021.

70. Li R, Wu K, Li Y, Liang X, Tse WKF, Yang L and Lai KP: Revealing the targets and mechanisms of vitamin $\mathrm{A}$ in the treatment of COVID-19. Aging (Albany NY) 12: 15784-15796, 2020.

71. Huda MN, Ahmad SM, Kalanetra KM, Taft DH, Alam MJ Khanam A, Raqib R, Underwood MA, Mills DA and Stephensen CB: Neonatal Vitamin A supplementation and Vitamin A status are associated with gut microbiome composition in Bangladeshi infants in early infancy and at 2 years of age. J Nutr 149: 1075-1088, 2019.

72. Murni IK, Prawirohartono EP and Triasih R: Potential role of vitamins and Zinc on acute respiratory infections including Covid-19. Glob Pediatr Heal 8: 1-8, 2021.

73. Iyer M, Jayaramayya K, Subramaniam MD, Lee SB, Dayem AA, Cho SG and Vellingiri B: COVID-19: An update on diagnostic and therapeutic approaches. BMB Rep 53: 191-205, 2020.

74. Brusselaers N: Prescribed drugs and the microbiome. Gastroenterol Clin North Am 48: 331-342, 2019.

75. Zeppa SD, Agostini D, Piccoli G and Stocchi V: Gut microbiota status in COVID-19: An unrecognized player? Front Cell Infect Microbiol 10: 576551, 2020.

76. Xu R, Lu R, Zhang T, Wu Q, Cai W, Han X, Wan Z, Jin X, Zhang $\mathrm{Z}$ and Zhang $\mathrm{C}$ : Temporal association between human upper respiratory and gut bacterial microbiomes during the course of COVID-19 in adults. Commun Biol 4: 24, 2021. 
77. Weersma RK, Zhernakova $\mathrm{A}$ and $\mathrm{Fu} \mathrm{J}$ : Interaction between drugs and the gut microbiome. Gut 69: 1510-1519, 2020.

78. Dormont F, Brusini R, Cailleau C, Reynaud F, Peramo A, Gendron A, Mougin J, Gaudin F, Varna M and Couvreur P: Squalene-based multidrug nanoparticles for improved mitigation of uncontrolled inflammation in rodents. Sci Adv 6: eaaz5466, 2020.

79. Liu L, Zheng Y, Yang J, Lv J and Fan C: Clinical observation on 13 novel coronavirus pneumonia cases treated with integrated traditional Chinese and Western Medicine. Zhejiang J Integr Tradit West Med 30: 349-351, 2020.

80. Dai T, Wang M, Wang P, Dai L, Dai R and Meng Q: Inhibition effects of eight anti-coronavirus drugs on glycosides metabolism and glycosidases in human gut microflora. Pharmazie 76 195-201, 2021.

81. Pan ZY, Chang YX, Han N, Hou FY, Lee BJY, Zhi FC, Yang RF and Bi YJ: Short-term high-dose gavage of hydroxychloroquine changes gut microbiota but not the intestinal integrity and immunological responses in mice. Life Sci 264 118450,2020

82. Million M, Lagier J, Gautret P, Colson P, Fournier PE, Amrane S, Hocquart M, Mailhe M, Esteves-Vieira V, Doudier B, et al: Early treatment of COVID-19 patients with hydroxychloroquine and azithromycin: A retrospective analysis of 1061 cases in Marseille France. Travel Med Infect Dis 35: 101738, 2020.

83. Parker EPK, Praharaj I, John J, Kaliappan SP, Kampmann B, Kang G and Grassly NC: Changes in the intestinal microbiota following the administration of azithromycin in a randomised placebo-controlled trial among infants in south India. Sci Rep 7: 9168,2017

84. Li R, Wang H, Shi Q, Wang N, Zhang Z, Xiong C, Liu J, Chen Y, Jiang $\mathrm{L}$ and Jiang Q: Effects of oral florfenicol and azithromycin on gut microbiota and adipogenesis in mice. PLoS One 12: e0181690, 2017.
85. Ried K, Travica N, Dorairaj R and Sali A: Herbal formula improves upper and lower gastrointestinal symptoms and gut health in Australian adults with digestive disorders. Nutr Res 76: 37-51, 2020.

86. Li Y, Liu X, Guo L, Li J, Zhong D, Zhang Y, Clarke M and Jin R: Traditional Chinese medicine for treating novel coronavirus (2019-nCoV) pneumonia: Protocol for a systematic review and meta-analysis. Syst Rev 9: 75, 2020.

87. de Munter J,Pavlov D, Gorlova A,Sicker M,Proshin A,Kalueff AV, Svistunov A, Kiselev D, Nedorubov A, Morozov S, et al: Increased oxidative stress in the prefrontal cortex as a shared feature of depressive- and PTSD-like syndromes: Effects of a standardized herbal antioxidant. Front Nutr 8: 661455, 2021.

88. Lyu M, Wang YF, Fan GW, Wang XY, Xu SY and Zhu Y: Balancing herbal medicine and functional food for prevention and treatment of cardiometabolic diseases through modulating gut microbiota. Front Microbiol 8: 2146, 2017.

89. Peterson CT, Sharma V, Uchitel S, Denniston K, Chopra D, Mills PJ and Peterson SN: Prebiotic potential of herbal medicines used in digestive health and disease. J Altern Complement Med 24: 656-665, 2018

90. Ma J, Zhou Q and Li H: Gut microbiota and nonalcoholic fatty liver disease: Insights on mechanisms and therapy. Nutrients 9: 1124,2017

91. Chen Z, Lv Y, Xu H and Deng L: Herbal medicine, gut microbiota, and COVID-19. Front Pharmacol 12: 646560, 2021

This work is licensed under a Creative Commons Attribution-NonCommercial-NoDerivatives 4.0 International (CC BY-NC-ND 4.0) License. 